\title{
High-pressure crystal structures of TaAs from first-principles calculations
}

\author{
Mingchun $\mathrm{Lu}^{1}$, Yanan $\mathrm{Guo}^{2 *}$, Miao Zhang ${ }^{3 *}$, Hanyu Liu ${ }^{2}$, John S. Tse ${ }^{2}$ \\ ${ }^{1}$ Department of Aeronautical Engineering Vocational Technology, Jilin University of \\ Chemical Technology, Jilin 132102, China \\ ${ }^{2}$ Department of Physics and Engineering Physics, University of Saskatchewan, \\ Saskatoon, Saskatchewan S7N 5E2, Canada \\ ${ }^{3}$ College of Physics, Beihua University, Jilin 132013, China \\ *Email: zhangmiaolmc@126.com (M. Zhang) ; yananguo813@gmail.com (Y. Guo)
}

\begin{abstract}
In this work, we systematically studied the phase transition of TaAs under high pressures and reported three high-pressure structures $P-6 m 2$ (hexagonal, stable at 13$32 \mathrm{GPa}$ ), $P 2{ }_{1} / c$ (monoclinic, stable at $32-103 \mathrm{GPa}$ ) and $P m-3 m$ (cubic, stable above $103 \mathrm{GPa}$ ), by using particle swarm optimization in combination with first principles electronic structure methodology. All predicted structures are dynamically stable, since there is no imaginary mode to be found in the whole Brillouin zone. At high pressures, the TaAs was found to become superconductor with the superconducting critical temperature of $\sim 1 \mathrm{~K}$ at about $100 \mathrm{GPa}$.
\end{abstract}

Key words: Wely semimetal; Structural prediction; High pressure;

\section{Introduction}

Weyl fermion was first predicted in 1929, it has not been observed in experiment. It possesses unique properties and can be used for quantum computers. ${ }^{1}$ Recently, it could be understood that a Weyl fermion can emerge as a quasiparticle in certain crystals, Weyl fermion semimetals. ${ }^{1-16} \mathrm{~A}$ Weyl semimetal is an unusual crystal where electrons behave as massless quasi-particle. Its electronic structure displays pairs of Weyl points connected by topological surface states, forming a unique arc-like Fermi surface.

1 
Recently, TaAs was identified to be the topological semimetal by first-principles calculations, which greatly facilitating an exploration of Weyl physics in real materials. ${ }^{17-20}$ However, the high-pressure research of TaAs is still scarce. At high pressure, the energy band gap of materials can overlap at sufficiently high pressure. Once these materials become good metal, they are possible to become good superconductors. A recent experimental work with Raman measurements ${ }^{21}$ suggested two possible phase transitions, and its theoretical predictions also suggest two candidate crystal structures. However, to best of our knowledge, there is no detailed study on superconductivity and crystal structures at ultra-high pressure.

In this work, we have extensively explored the high-pressure phases of TaAs by using extensive structure searching in conjunction with first-principles calculations. A novel hexagonal structure of $P-6 m 2$ was found to be stable at $13 \mathrm{GPa}$. At higher pressure, a monoclinic $P 2{ }_{1} / c$ is more stable than $P-6 m 2$ at $32 \mathrm{GPa}$. At $103 \mathrm{GPa}$, the enthalpy of a cubic Pm-3m structure becomes more favorable. All predicted structures are calculated to show dynamically stable. Furthermore, the superconductivity is examined. The calculated superconducting critical temperature of TaAs reaches $1 \mathrm{~K}$ at about $100 \mathrm{GPa}$.

\section{Methods}

Our approach is based on a global minimization of free energy surfaces via PSO technique as implemented in CALYPSO (crystal structure AnaLYsis by particle swarm optimization) code, ${ }^{22,23}$ which was unbiased by any prior known structures. The approach has correctly predicted the crystal structures of a diverse variety of materials. ${ }^{24-29}$ The underlying ab initio structure relaxation was performed using density-functional theory within projector augmented wave method as implemented in the Vienna Ab-inito Simulation Package code (VASP). ${ }^{30-32}$ The projector augmented wave (PAW) potentials ${ }^{33}$ used here were derived using the generalized gradient approximation (GGA) ${ }^{34}$ functional with valence electrons of $5 d^{3} 6 s^{2}$ and $4 s^{2} 4 p^{3}$ for Ta and As, respectively, suitable for the high-pressure study. A plane-wave kinetic energy 
cutoff of $800 \mathrm{eV}$ and the use of Monkhorst-Pack $k$-points meshes of $12 \times 12 \times 12,6 \times$ $5 \times 6,16 \times 16 \times 16$, and for $P-6 m 2, P 2_{1} / c$ and $P m-3 m$ in the Brillouin zones produced enthalpy results well converged to below $1 \mathrm{meV} / \mathrm{atom}$, respectively. The electronphonon coupling properties were examined using linear response theory as implemented in Quantum Espresso package. ${ }^{35}$ A MP grid of $16 \times 16 \times 16$ for Pm-3m was used to ensure k-point sampling convergence with Gaussians of width $0.03 \mathrm{Ry}$, which approximates the zero-width limits in the calculations of electron-phonon coupling constant parameter $\lambda$.

\section{Results and discussion}

To explore crystal structures of TaAs at high pressures, we have performed in the pressure range 0-120 GPa with 1-4 f.u. per simulation cell. At ambient pressure, our structure predictions successfully reproduced the experimental $I 4_{1} m d$ structure. At higher pressure, our structural searches identified three high-pressure structures with $P-6 m 2, P 2{ }_{1} / c$ and $P m-3 m$ space groups at different pressures, respectively. The structural parameters of these three phases are listed in Table 1. It is noteworthy that $P-6 m 2, P 2{ }_{1} / c$ phases are also predicted by recent theoretical work. ${ }^{21}$ It is interesting to see that the coordination of Ta increased from $6(P-6 m 2)$ to $7\left(P 2_{1} / c\right)$ and then to 8 $(P m-3 m)$ as shown in Figure 1. Moreover, it suggests that TaAs can adopt a dense cubic packing structure at sufficiently high pressure. The enthalpy $H=U+P V$ determined the stability of different phases at $0 \mathrm{~K}$. As calculations suggested, the cubic phase possesses the smallest volume (24.47 $\AA^{3 / f}$.u.) compared to the $P-6 m 2$ (31.92 $\AA^{3} /$ f.u.) and $P 2{ }_{1} / c\left(29.01 \AA^{3} /\right.$ f.u. $)$ phases.

Furthermore, we examined the enthalpies of the newly predicted stable phases which are plotted as a function of pressure in Figure 2 to compare with the experimental $I 4_{1} m d$ structure. It clearly shows that this $I 4{ }_{1} m d$ structure is the most stable phase up to $13 \mathrm{GPa}$, beyond which our hexagonal $P-6 m 2$ structure becomes most favorable until to $32 \mathrm{GPa}$. In the pressure range of 32-103 GPa, the monoclinic $P 2{ }_{1} / c$ structure becomes the most stable phase. At higher pressure (above $103 \mathrm{GPa}$ ), 
the enthalpy of cubic Pm-3m structure begins to become more favorable. In order to examine the dynamical stability, we calculated phonon dispersion curves for these three high-pressure structures using the supercell method as implanted in PHONOPY software package. No imaginary phonon frequencies are found in their low-enthalpy pressure range in the whole Brillouin zone (Figure 3), indicating that they are dynamically stable phases.

Previous experiment and theory suggest TaAs is a weyl semimetal. There are "Weyl cones" and "Weyl nodes" to be found at around bulk or surface electronic band structures. The pressure is well known to alter chemical bonding environment and may significantly revise the electronic band structures. Therefore, it is important to explore the evolution of electronic structure for TaAs at high pressures. In Figure 4 (a)-(c), we have calculated electronic band structures of TaAs in the absence of spinorbit coupling for $P-6 m 2, P 2{ }_{1} / c$ and $P m-3 m$ structures at20, 40 and $110 \mathrm{GPa}$, respectively. It is noteworthy that TaAs still has a band touch point along some direction (Figure $4 \mathrm{a}$ and $4 \mathrm{~b}$ ) at high pressures. For instance, there is a semimetal point for $P-6 m 2$ phase along $\mathrm{K}-\Gamma$ direction and for $P 2_{1} / c$ structure along $\Gamma-\mathrm{Y}$ direction. However, at higher pressure, TaAs becomes a good metal due to the strong overlap between conduction and valence bands. It is found that many bands crossed the Fermi energy. At some directions $(\mathrm{X}-\mathrm{R}$, around $\Gamma$ and around $\mathrm{M})$, it is clearly seen that there are quadratic type bands in band structure (Figure 4c). This feature suggests TaAs may become a good superconductor at high pressure. It is well known that the spin-orbital coupling effect in TaAs is very important for its electronic structure. We have studied the spin-orbit coupling (Figure $4 \mathrm{~d}-\mathrm{f}$ ). It is clearly seen that some bands in TaAs are split.

In previous study, it is suggested a high-purity sample of tantalum possess superconductivity. ${ }^{36}$ TaAs may become a superconductor since it is a good metal in electronic calculation. To explore the potential superconductivity, we have thus performed the electron-phonon coupling calculations on TaAs at high pressure based on linear response theory as implanted in Quantum Espresso package. ${ }^{35}$ In Figure 5, it 
shows the calculated electron-phonon coupling parameter $\lambda$, averaged frequency $W_{l o g}$ and superconducting critical temperature $T_{c}$ as a function of pressure. The $T_{c}$ was estimated based on the Allen-Dynes modified McMillan equation, ${ }^{37}$ where $\mu^{*}$ is the Coulomb pseudopotential. At $40 \mathrm{GPa}$, TaAs does not show the superconductivity, due to its small $\lambda(0.31)$. As shown in Fig. 4b, there are not many bands to cross Fermi energy. This indicates the weak electron-phonon coupling strength, since strong electron-phonon coupling needs more electrons at Fermi energy level to couple with an approximate phonon medium. In Figure $4 b$, more bands were found to cross the Fermi energy and strong band overlap suggest stronger electron-phonon coupling strength compared to those of low pressures phases. At $100 \mathrm{GPa}$, the calculated $\lambda$ is 0.44 and the $T_{c}$ of TaAs reach $1 \mathrm{~K}$ with the typically Coulomb pseudopotential of $0.13 .^{38-40}$ It is found that the logarithmic average of the phonon frequency increased with pressure as expected. However, the electron-phonon calculations suggest the calculated $T_{c}$ of TaAs decreased above $100 \mathrm{GPa}$ due to decreased $\lambda$. Finally, we should point out that we did not consider spin-orbital effects in electron-phonon coupling calculations. It is well known that spin-orbital effects lead to band splitting for heavy elements. As shown in Fig. 4c and 4f, there are more parallel bands around Fermi level in band structures with considering spin-orbital effects, indicating stronger Fermi surface nesting ${ }^{41}$ that thus enhanced the superconductivity of TaAs. Since the electron-phonon coupling calculation with considering spin-orbital effects is time demanding, it should be considered in the future work and beyond the scope of this study.

\section{Conclusion}

In this work, we systematically explored crystal structures of TaAs under high pressures using recently developed particle swarm optimization mythology. In our structure searches, we found that three high-pressure structures P-6m2 (hexagonal, stable at 13-32 GPa), $P 2_{1} / c$ (monoclinic, stable at 32-103 GPa) and Pm-3m (cubic, stable above $103 \mathrm{GPa}$ ). Phonon calculations suggest these structures are dynamically 
stable. In electronic calculations, the TaAs still shows band touch feature at high pressure. At higher pressure, the cubic phase of TaAs shows the good metal feature. The electron-phonon coupling calculations indicate the superconducting critical temperature reaches $\sim 1 \mathrm{~K}$ at about $100 \mathrm{GPa}$.

\section{Acknowledgements}

M. Z. acknowledges funding support from the National Natural Science Foundation of China under Grants No. 11504007. J.S.T. and H.L. acknowledge the National Science Foundation of CHINA (11474126) and support from the University of Saskatchewan research computing group and the use of the HPC resources (Plato machine). H.L. also acknowledge the XSEDE computer resource.

\section{References}

1 H. Z. Weyl, Phys 56 (1929) 330.

2 S. Xu, I. Belopolski, N. Alidoust, M. Neupane, G. Bian, C. Zhang, R. Sankar, G.

Chang, Z. Yuan, C. Lee, S. Huang, H. Zheng, J. Ma, D. Sanchez, B. Wang, A. Bansil,

F. Chou, P. P. Shibayev, H. Lin, S. Jia, M. Hasan, Science 349 (2015) 613.

3 F. Wilczek, Phys. Today 51 (1998) 11.

4 L. Balents, Physics 4(2011) 36.

5 H. B. Nielsen, M. Ninomiya, Phys. Lett. B 130 (1983) 389.

6 X. Wan, A. M. Turner, A. Vishwanath, S. Y. Savrasov, Phys. Rev. B 83 (2011) 205101.

7 B. Singh et al., Phys. Rev. B 86 (2012) 115208.

8 S.-Y. Xu et al., Science 332 (2011) 560-564.

9 S.-M. Huang et al., Nat. Commun. 6 (2015)7373.

10 H. Weng, C. Fang, Z. Fang, F. B. Andrei Bernevig, X. Dai, Phys. Rev. X 5 (2015) 011029.

11 J. Liu, D. Vanderbilt, Phys. Rev. B 90 (2014), 155316.

12 A. A. Zyuzin, A. A. Burkov, Phys. Rev. B 86 (2012) 115133.

13 D. T. Son, B. Z. Spivak, Phys. Rev. B 88 (2013) 104412.

14 C.-X. Liu, P. Ye, X.-L. Qi, Phys. Rev. B 87 (2013) 235306. 
15 H. Wei, S.-P. Chao, V. Aji, Phys. Rev. Lett. 109 (2012) 196403.

16 P. E. C. Ashby, J. P. Carbotte, Phys. Rev. B 87 (2013) 245131.

17 S.-Y. Xu, et al., Science 349 (2015) 613.

18 L. Yang, et al., Nat. Phys. 11 (2015) 728.

19B. Lv, et al., arXiv preprint arXiv:1503.09188 (2015).

20 B. Lv, et al., Phys. Rev. X 5 (2015) 031013.

21 Y. Zhou, et al., arXiv preprint arXiv:1509.07361 (2015).

22 Y. Wang, J. Lv, L. Zhu, and Y. Ma, Comput. Phys. Commun. 183 (2012) 2063.

23Y. Wang, J. Lv, L. Zhu, and Y. Ma, Phys. Rev. B 82 (2010) 094116.

24 J. Lv, Y. Wang, L. Zhu and Y. Ma, Phys. Rev. Lett. 106 (2011) 15503.

25 L. Zhu, H. Wang, Y. Wang, J. Lv, Y. Ma, Q. Cui and G. Zou, Phys. Rev. Lett. 106 (2011) 145501.

26 M. Zhang, Hanyu Liu, Q. Li, B. Gao, Y. Wang, H. Li, C. Chen, and Y. Ma, Phys. Rev. Lett. 114 (2015) 015502.

27 M. Zhang, H. Liu, Y. Du, X. Zhang, Yanchao Wang, Quan Li, Phys. Chem. Chem. Phys. 15 (2013) 14120.

28 M. Zhang, G. Gao, A. Kutana, Y. Wang, X. Zou, J. S. Tse, B. I. Yakobson, H. Li, H. Liu, and Y. Ma, Nanoscale, 7 (2015) 12023.

29 Q. Li, D. Zhou, W. Zheng, Y. Ma and C. Chen,Phys. Rev. Lett. 110, 136403 (2013).

30 G. Kresse and J. Furthmüller, Phys. Rev. B 54 (1996) 11169.

31 G. Kresse and J. Hafner, Phys. Rev. B 49 (1994) 14251.

32 G. Kresse and J. Hafner, Phys. Rev. B 47 (1993) 558.

33 P. E. Blochl, Phys. Rev. B 50 (1994) 17953.

34 J. P. Perdew, K. Burke, and M. Ernzerhof, Phys. Rev. Lett. 77 (1996) 3865.

35 P. Giannozzi, S. Baroni, N. Bonini, M. Calandra, R. Car, C. Cavazzoni, and D.

Ceresoli, J. Phys. Condens. Matter 21 (2009) 395502.

36 J. Milne, Physical Review 122 (1961) 387.

37 R. Dynes, Solid State Commun. 10 (1972) 615.

38 Y. Li, J. Hao, H. Liu, Y. Li, and Y. Ma, J. Chem. Phys 140 (2014) 174712. 
39 D. Duan, et al., Sci. Rep. 4 (2014) 6968.

40 N. Ashcroft, Phys. Rev. Lett. 92 (2004) 187002.

41 J. S. Tse, Y. Yao and K. Tanaka, Phys. Rev. Lett. 98 (2007) 117004. 
Table 1 Calculated lattice constants $(\AA)$, atomic positions and bond lengths for $P-6 m 2$, $P 2{ }_{1} / c$ and $P m-3 m$ phases at ambient pressure.

\begin{tabular}{|c|c|c|c|}
\hline Structure & $P-6 m 2$ & $P 2_{1} / c$ & $P m-3 m$ \\
\hline Lattice constants & $a=3.409$ & $a=6.141, b=4.896$ & $a=3.212$ \\
\hline$(\AA)$ & $c=3.488$ & $c=6.221, \beta=130^{\circ}$ & \\
\hline \multirow{5}{*}{ Atom positions } & $\mathrm{Ta}(0.667,0.333,0)$ & Тa $(0.816,0.144,0.708)$ & $\mathrm{Ta}(0,0,0)$ \\
\hline & As $(0.333,0.667,0.5)$ & As $(0.309,0.354,0.226)$ & As $(0.5,0.5,0.5)$ \\
\hline & & Ta-As1 2.714 & \\
\hline & & Ta-As2 2.717 & \\
\hline & & Ta-As3 2.762 & \\
\hline \multirow[t]{4}{*}{ Bond lengths $(\AA)$} & Ta-As 2.629 & Ta-As4 2.647 & Ta-As 2.781 \\
\hline & & Ta-As5 2.715 & \\
\hline & & Ta-As7 2.646 & \\
\hline & & Ta-As7 3.384 & \\
\hline
\end{tabular}




\section{Figure captions:}

Figure 1. Predicted TaAs structures. (a) $P-6 m 2$, (b) $P 2{ }_{1} / c$ and (c) $P m-3 m$ phase. The yellow brown and green spheres represent Ta and As atoms, respectively.

Figure 2. Calculated enthalpies per formula unit as functions of pressure between 0 and $120 \mathrm{GPa}$ with respective to $I 4_{1} m d$ structure. Inset shows enthalpy versus pressure for $P-6 m 2$ structure relative to that of the $I 4_{1} m d$ structure.

Figure 3. Phonon dispersion curves of (a) $P-6 m 2$, (b) $P 2_{1} / c$ and (c) $P m-3 m$ structures of TaAs at 20, 40 and $110 \mathrm{GPa}$, respectively.

Figure 4. Electronic properties of (a) $P-6 m 2$, (b) $P 2_{1} / c$ and (c) $P m-3 m$ structures of TaAs in the absence of spin-obit coupling from DFT at 20, 40 and $110 \mathrm{GPa}$, respectively. (d)-(f) The same as panel (a)-(c) in the presence of spin-orbit coupling.

Figure 5. The calculated superconductivity of TaAs at high pressure. 

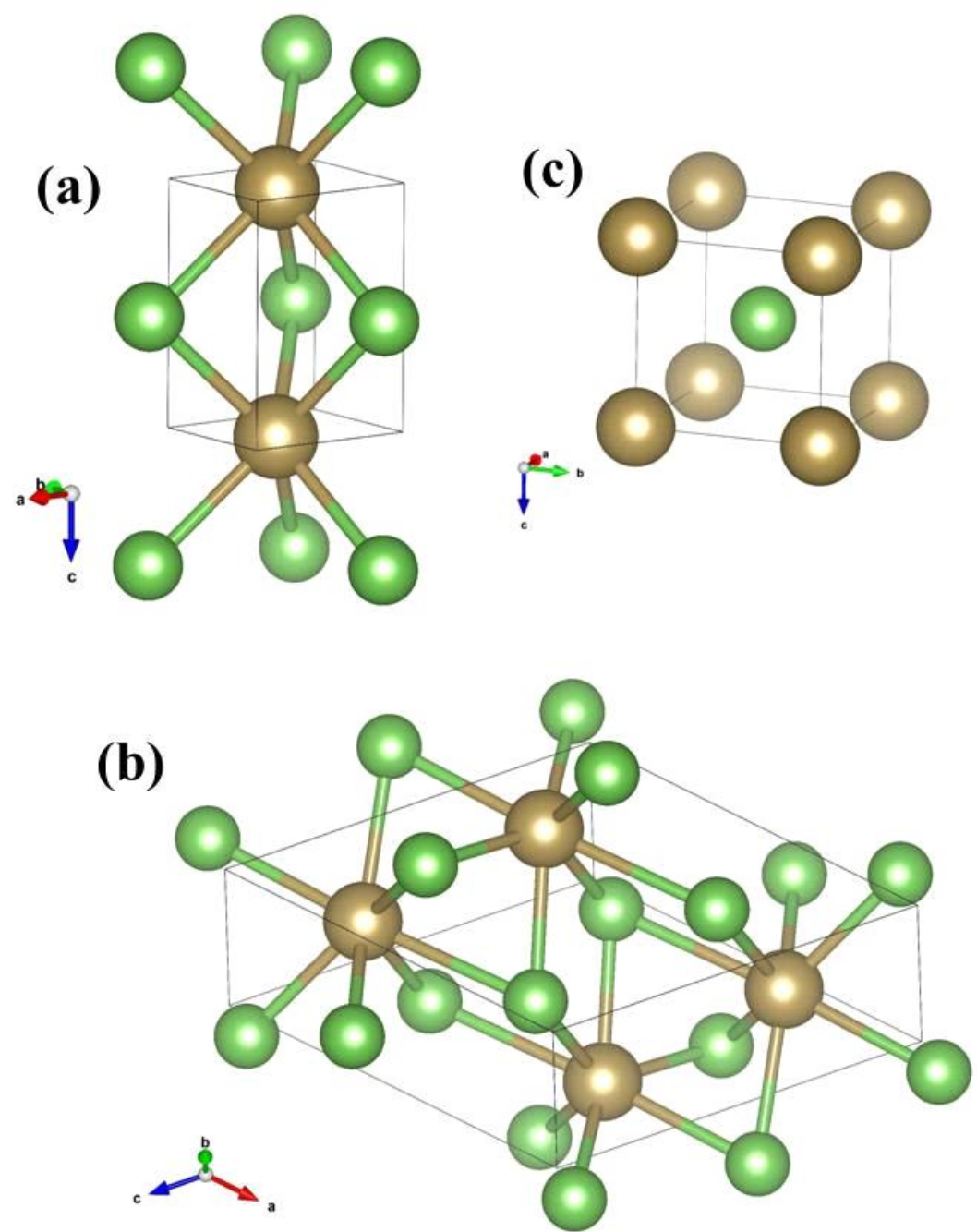

Figure 1 


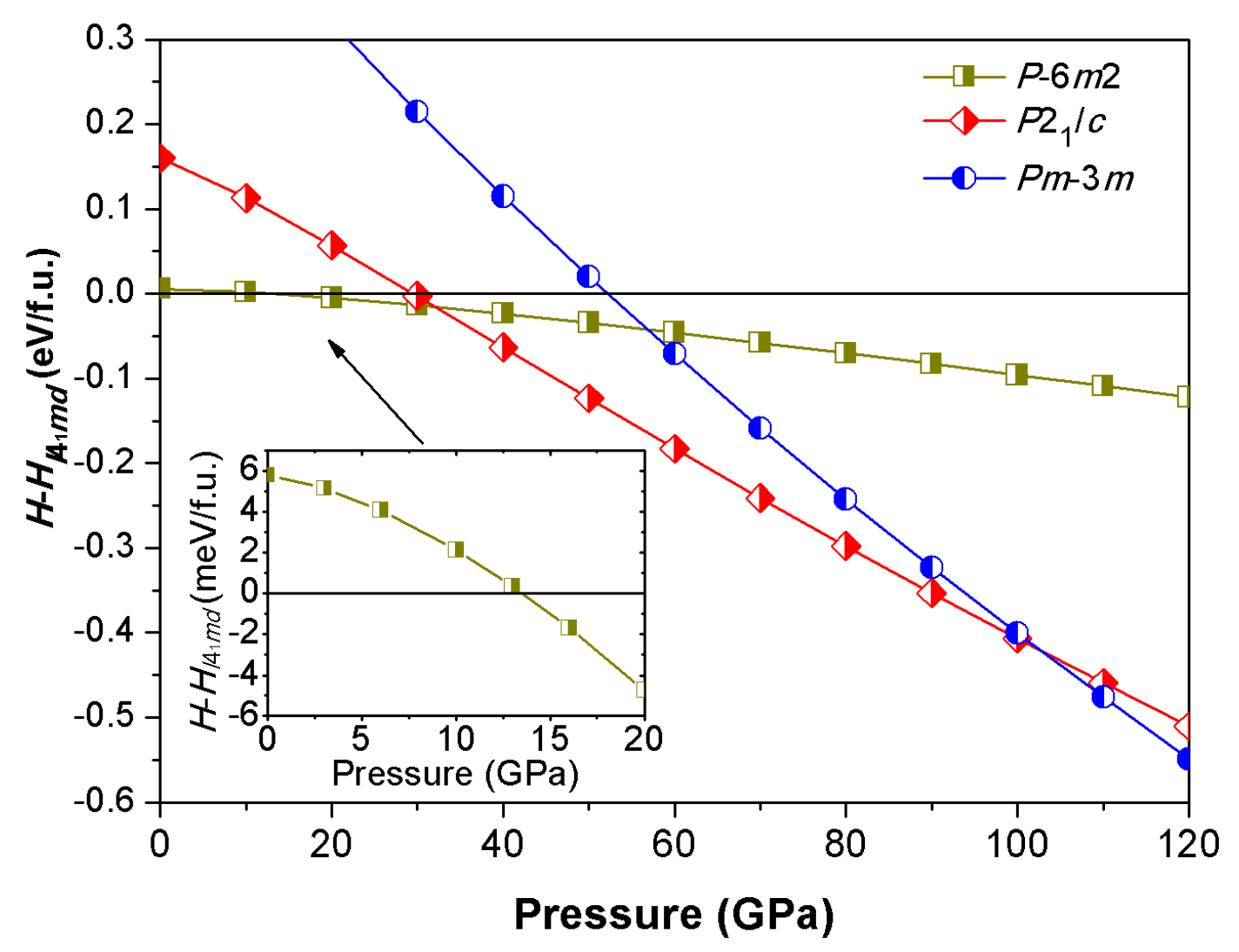

Figure 2 

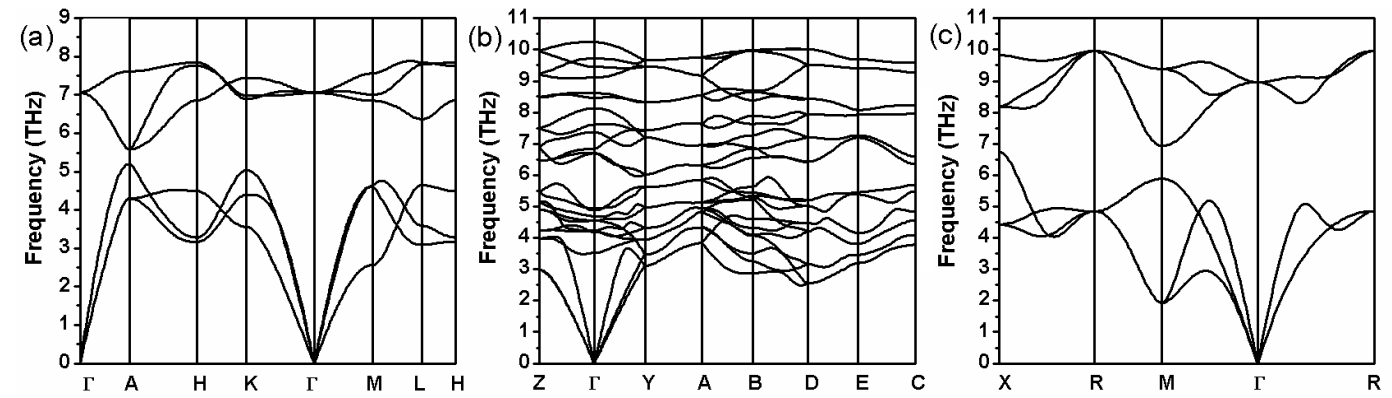

Figure 3 

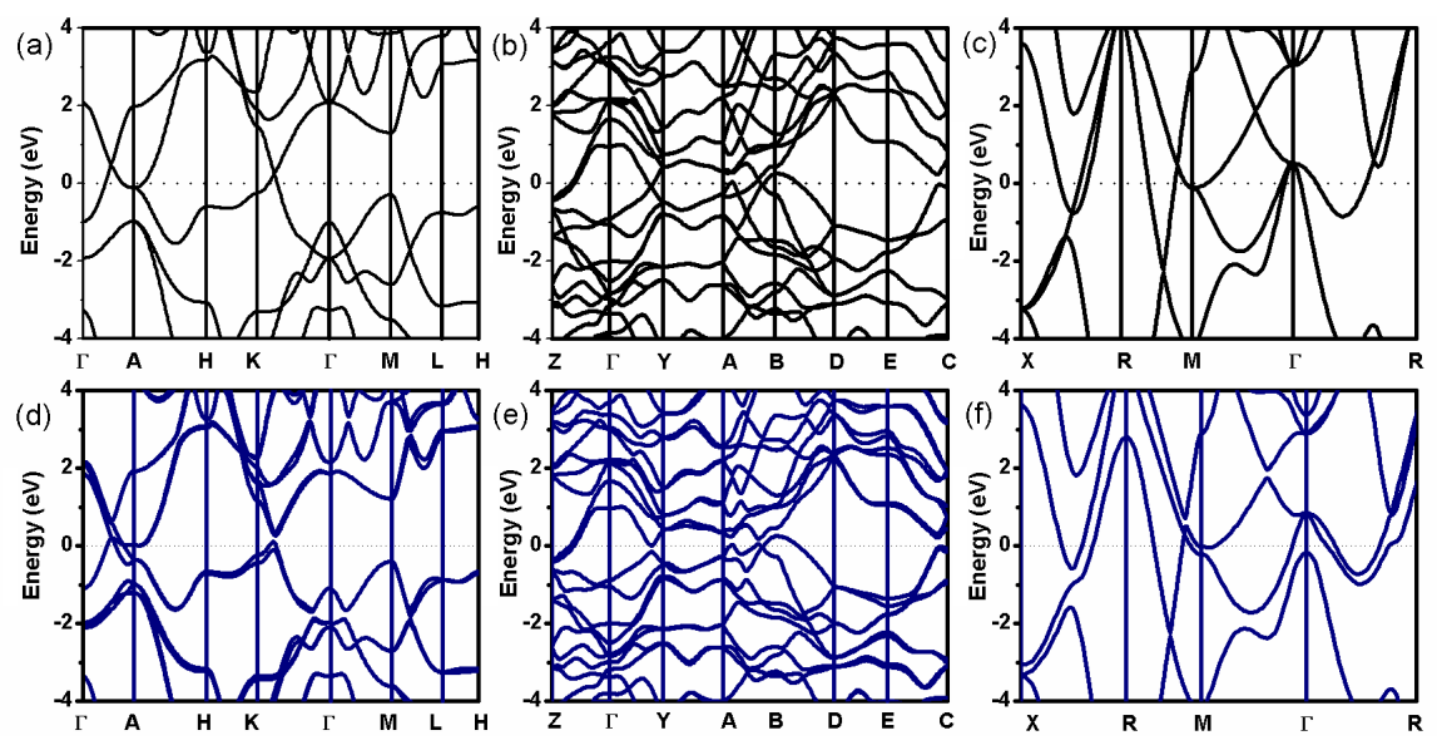

Figure 4 


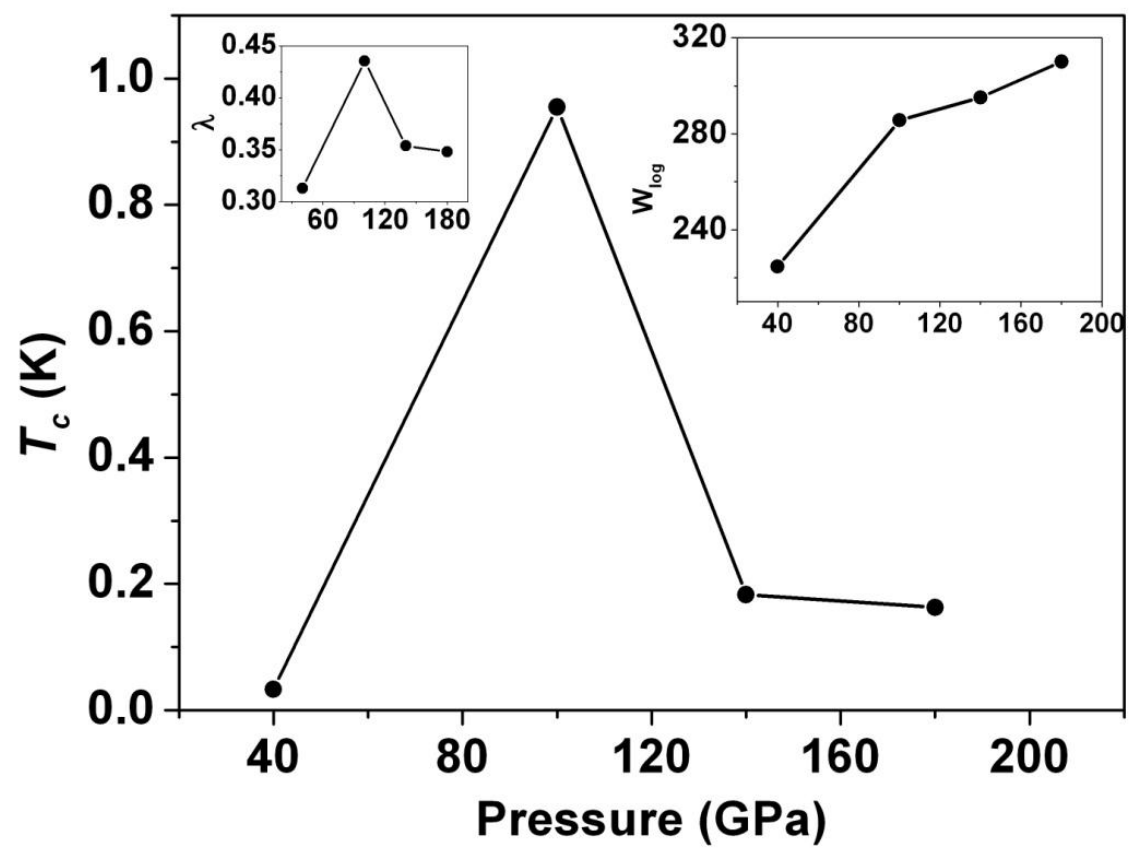

Figure 5 